\title{
Método TRADIN: transparência governamental com a cooperação da economia digital
}

\author{
Giovanni C. Biffi ${ }^{1}$, Eunice P. Santos Nunes ${ }^{2}$, Cristiano Maciel $^{2}$ \\ ${ }^{1}$ tradin.org \\ Av. República do Líbano, 1798 - Cuiabá/MT - Brasil \\ ${ }^{2}$ Instituto de computação - Laboratório de Ambientes Virtuais Interativos (LAVI) \\ Universidade Federal de Mato Grosso (UFMT) - Cuiabá/MT \\ \{gantesbr, eunice.ufmt, crismac\} @gmail.com
}

\begin{abstract}
Public authorities have been moving towards government transparency. However, open data laws and principles, which take into account citizens' interests and needs, are being neglected in the publishing of government information. The cooperation of the digital economy points the way to the efficiency of government transparency. In this scenario, the method TRADIN (Method for Transparent, Dynamic and Integrated Information Publishing) is herein presented. It provides society with standardized open data, thus enabling the development of integrated services and / or applications.
\end{abstract}

Resumo. Os poderes públicos têm realizado movimentos em direção a transparência governamental. Contudo, a observância das leis e princípios de dados abertos, que levem em conta os interesses e necessidades do cidadão, está sendo deixada de lado quando se faz a publicação de informações governamentais. A cooperação da economia digital aponta o caminho para a eficiência da transparência governamental. Nesse cenário, este trabalho apresenta o método TRADIN (Método para Publicação Transparente, Dinâmica e Integrada de Informações), que propicia dados abertos de forma padronizada para a sociedade, possibilitando novas produções de serviços e/ou aplicativos integrados.

\section{Introdução}

No Brasil, o controle social dos atos do administrador público está previsto em vários dispositivos constitucionais. Conforme Art. 5º, inciso XXXIII da Constituição Federal do Brasil (1988), "todos têm o direito de receber informações das esferas públicas para o seu uso particular e/ou coletivo, observando-se a legislação vigente e o caráter sigiloso das informações em relação à segurança da sociedade e do Estado brasileiro". Deste modo, Rek (2013) declara que a sociedade deve estar ciente da subordinação da Administração Pública aos princípios que a regem, atuando inclusive como fiscais da "res" pública, utilizando-se dos meios legais e alternativos disponíveis para denúncias e repúdios de corrupção, fraudes e abusos. 
As instituições governamentais brasileiras devem permitir o acesso universal às informações, de acordo com a Lei da Transparência $n^{0}$ 131/2009 e a Lei de Acesso a Informação $n^{0} 12.527 / 2011$. Não obstante, existem diversas problemáticas, não somente técnicas, mas também éticas e jurídicas, a respeito das propriedades intelectuais, segurança da informação e direito de publicidade de conteúdos. Ressalta-se que uma parte das informações divulgadas nos sítios governamentais não está de acordo com os anseios da sociedade [Araújo; Maia 2014], e não proporcionam a reutilização das informações [Martano; Craveiro 2014]. De acordo com pesquisa sobre Transparência Governamental, nos portais da transparência ocorre um baixo alcance nos níveis de maturidade da transparência, os quais se restringem apenas em atender as conformidades recentes da legislação brasileira [Cappelli et al. 2014].

Os conteúdos disponibilizados nos sítios governamentais precisam prever a transparência dos dados, cujas peculiaridades devem atender a integrabilidade, interoperabilidade, acessibilidade e responsividade, provendo assim serviços que contemplam o cidadão com a ampliação de informações transparentes, eficientes e democráticas [Espíndola; Oliveira; Formiga 2011]. O governo, observando suas limitações, deve prover serviços, disponibilizando dados primários a serem explorados também por outros órgãos públicos e pelas iniciativas privadas. Estas devem criar interações que satisfaçam a sociedade atendendo seus desejos, anseios e necessidades, impulsionando um novo modelo econômico pela internet, a economia digital.

No entanto, são encontradas algumas problemáticas em relação a facilidade de reutilização dos dados, a confiabilidade das informações publicadas e o compartilhamento das informações, de forma automatizada [Bargh, Choenni e Meijer 2015]. Destacamos que a reutilização dos dados pode auxiliar na fiscalização dos recursos públicos, analisar as deficiências na administração pública e melhorar o controle da corrupção.

Por isso, Collin-Williams e Wolfe (2010) investigam como a World Trade Organization (WTO) usa a transparência como instrumento de política comercial. Sugerem que transparência reduz as incertezas e a política obtém a confiança necessária de investidores para tomada de decisões nos investimentos. Contudo, a transparência deve ser uma força de reforma, e a informação deve ser analisada e estar amplamente disponível para aqueles que podem influenciar os governos.

Em busca de soluções tecnológicas que melhorem a percepção dos cidadãos em relação a aplicação dos recursos públicos, o compartilhamento de informações precisa considerar além da transparência, sua manutenção e expansão acerca da reutilização das informações [Santos et al. 2016]. Deste modo, há indícios de que a transparência pode acontecer com melhor eficiência se houver a parceria público-privado, ou seja, se a transparência governamental for inserida na economia digital, impactando diretamente na tomada de decisão dos cidadãos.

Nesse cenário, este estudo propõe um método que contempla a publicação dinâmica de informações democráticas e busca a possibilidade de diálogo com $o$ ambiente externo à organização, a fim de alcançar melhores níveis de maturidade em transparência organizacional e estimular a economia digital. Logo, o objetivo deste estudo é apresentar a concepção do método TRADIN, um método para publicação transparente, dinâmica e integrada de informações, integrando as múltiplas fontes de 
sistemas de informações internas dos órgãos de governo, a fim de viabilizar o uso destas por outros órgãos governamentais ou outros segmentos da sociedade, de modo transparente, dinâmico, distribuído e integrado.

\section{Metodologia}

A metodologia empregada nesta pesquisa teve início com uma revisão bibliográfica, a fim de responder a questão de pesquisa "Quais são os protocolos e principais tecnologias disponíveis que estabelecem um ambiente propício para divulgação e reutilização das informações públicas?”. A revisão bibliográfica foi realizada aplicandose strings de busca, combinadas a partir das palavras-chave definidas, nas bases de dados IEEE, ACM, Google Scholar e em anais de conferências da área.

$\mathrm{Na}$ sequência, foi elaborado um Mapa Mental, com os principais aspectos do problema. O Mapa Mental buscou compreender quais são as dificuldades e necessidades dos órgãos governamentais e dos ambientes externos em relação à transparência dos conteúdos disponibilizados. O Mapa Mental proporciona benefícios, os quais se sobressaem a organização das ideias e conceitos, pois destacam-se palavras-chave relevantes, agrupam ideias e estimulam a criatividade e inovação [Batista; Silva 2015].

Considerando a importância da integração entre o órgão governamental e o ambiente externo, foi desenvolvido o método TRADIN, suportado por uma ferramenta com o mesmo nome, fazendo uso de diferentes tecnologias. Definiu-se a estruturação das saídas em HTML (HyperText Markup Language), RDF (Resource Description Framework) e JSON (JavaScript Object Notation), como os principais formatos de saída gerados pelos sistemas.

O método de pesquisa qualitativo abordado por Stake (2011) foi adotado para descrever como os objetivos propostos foram alcançados, descrevendo a arquitetura do ambiente, as técnicas de padronização na manutenção dos registros e a comunicação entre os ambientes, de forma integrada.

\section{Representação da informação na Internet}

A transparência acontece quando o Estado, em seu sentido mais amplo, está aberto e visível ao cidadão [Klein, Luciano e Macadar 2015]. A explicitação de informações, realizada com prontidão e confiabilidade pelo Estado para cidadão, exibindo de modo coeso o que acontece em seu ambiente de competência, é considerada transparência.

Publicar informações com representação padronizada e utilizar tecnologias que permitam sua análise e absorção pelos cidadãos, são questões consideradas relevantes para transparência das informações nos órgãos governamentais. Assim, apresentar os protocolos e principais tecnologias que podem estabelecer um ambiente propício para divulgação e reutilização das informações públicas é indispensável.

Ademais, Washington, Willis e Tauberer (2012) afirmam que as mudanças feitas nos sítios de governo ocorrem lentamente e existem limitações na busca. Os autores debatem o modelo público-privado e discutem opiniões sobre atores nãogovernamentais que fornecem informações públicas gratuitas e listam os benefícios e desafios de adequar a informação governamental à economia digital. 
Em setembro de 2011, o governo brasileiro assinou a Declaração do Governo Aberto, na qual se comprometeu em apresentar planos de ação para atender as expectativas cívicas dos brasileiros, prover o crescente acesso à informação e divulgar suas atividades em todos os níveis dos órgãos públicos. Por meio dos Dados Abertos Governamentais, é possível difundir e incentivar hábitos relacionados à transparência dos dados, à participação social e ao acesso à informação pública. Dados providos pelos órgãos públicos, de forma livre, podem ser utilizados por iniciativas privadas da sociedade civil ou introduzidos em novas produções de serviços e/ou aplicativos em diferentes órgãos de governo [Klein et al. 2015].

O Modelo de Maturidade em Transparência proposto por Cappelli et al. (2014) contempla cinco níveis de transparência organizacional (1. Opaca, 2. Divulgada, 3. Compreendida, 4. Confiável, 5. Examinável). O objetivo do modelo é orientar as organizações no alcance do nível de transparência desejado, seja para informações quanto para processos. No entanto, Cappelli et al. (2014) destacam que diversos portais governamentais estagnaram no Nível 2, da informação divulgada, o que não garante que a informação será entendida, confiável e examinável, o que pode gerar o desinteresse da sociedade. Os autores salientam que são necessários investimentos e empenho das organizações em alcançar o nível "Examinável” (Nível 5), no qual é possível que as informações publicadas pelas organizações sejam examinadas, auditadas e verificadas completamente pelo ambiente externo a organização.

Salienta-se que os níveis de Maturidade de Transparência Organizacional apresentados por Cappelli et al. (2014) se relacionam com os oito princípios de DGA Dados Governamentais Abertos [Davies 2013], os quais são amplamente aceitos pela comunidade mundial, ou seja, dados abertos têm origem em dados completos, primários, atuais, acessíveis, compreensíveis por máquina, não discriminatórios, não proprietários e de licenças livres.

Destaca-se que é importante buscar características técnicas que padronizem os sítios governamentais, facilitando o acesso à informação. No atual cenário tecnológico, encontra-se uma baixa qualidade dos dados disponibilizados, a não observância das leis e princípios dos dados abertos, a baixa frequência das atualizações das informações, a falta de manutenção das informações e a diversidade de conhecimentos requeridos pelas equipes para atuar nesta área [Cruz et al. 2016].

Mendonça, Maciel e Viterbo Filho (2015) apresentam a arquitetura para a publicação de dados abertos, compostos de sete etapas: obter dados, criar banco de dados relacional, criar ontologias, criar triplas RDF, importar triplas para banco de dados, fornecer triplas em ARC 2 Servidor e exibição em um mashup. Os passos para a publicação de dados abertos requerem um único banco de dados relacional, cuja criação ocorre por meio da convergência de bases de dados dos departamentos estaduais e municipais de saúde de Cuiabá, no estado de Mato Grosso. A segunda etapa (criar base relacional) definida no método de Mendonça, Maciel e Viterbo Filho (2015) é questionável se comparada ao segundo princípio definido na DGA, no qual é considerada a publicação de dados primários, sem agregação e com granularidade. A fusão dos dados de duas secretarias na criação de uma única base relacional retira a primariedade esperada dos dados publicados, considerando que os dados originários das 
duas secretarias deveriam ser analisados individualmente, para manter-se a primariedade.

De forma mais complexa, Paiva, Revoredo e Baião (2016) apresentam uma arquitetura que propõe uma solução para integração de diversas fontes para a publicação de informações da transparência. Nessa arquitetura, também apoiada em banco de dados, é possível fazer a publicação das informações de transparência. São apresentadas seis etapas, nas quais são realizadas atividades para a publicação das informações, e estas etapas são compreendidas por: ETL (Extract, transform, load), DW (Data Warehouse), DM (Data Mart) Portal / Gestores do Portal, Painel de Controle do DW, BDs (Data Bases) Site e Data Marts de outras áreas. No entanto, apresenta limitações em relação a primariedade dos dados publicados, devido a junção de diversas fontes para a criação de uma única fonte de dados para transparência. Nesta arquitetura de solução, existem dois fatores controversos aos princípios estabelecidos na DGA. Em relação ao primeiro fator, observa-se na Etapa 1, compreendida por ETL, na qual acontece a conversão das fontes para um único DW, ocorre a agregação e também a perda de granularidade dos dados por meio dos fatores de conflitos de integração dos dados do Portal da Transparência relacionados por Paiva, Revoredo e Baião (2016). Outro fator polêmico, é a atuação dos gestores do DW, prevista nas Etapas 3 e 4, DW (Data Warehouse), DM (Data Mart) Portal / Gestores do Portal respectivamente, na qual pessoas gerenciam os dados para publicação. Dessa forma, pode acontecer a incompletude das informações públicas, pois se considera a publicação das informações mais convenientes para o órgão, o que pode ocasionar falhas na interpretação do caráter sigiloso das informações no momento da publicação.

Nota-se que as arquiteturas de publicação de dados abertos propostas por Mendonça, Maciel e V. Filho (2015) e Paiva, Revoredo e Baião (2016), respectivamente, atendem a publicação das informações de transparência, no entanto, focaram suas bases na junção dos bancos de dados das diversas fontes de informação, para em seguida aplicar os tratamentos necessários para publicação, propiciando espaço para surgirem problemas como, por exemplo, baixa velocidade de publicação, centralização de esforços e qualidade das informações disponibilizadas. Isto nos levou a uma reflexão sobre a exiguidade de um método que não focasse no banco de dados, como sugerido por Paiva, Revoredo e Baião (2016), mas sim na consulta dos dados.

Assim, observamos a possibilidade de utilizar os objetos de consulta providos pelos sistemas primários ou arquivos de dados gerados por estes, nos dados convertidos direto da fonte primária em arquivos RDF (Resource Description Framework) e JSON (JavaScript Object Notation), que poderão ser exibidos nas páginas de transparência dos sítios por meio da construção de requisições HTTP (Hypertext Transfer Protocol), principal protocolo de comunicação da internet.

No protocolo HTTP, trafega a linguagem de marcação interpretada pelos navegadores após o término da requisição, o HTML. Os sítios de transparência devem apresentar um padrão na montagem das páginas que os compõem, no entanto, as páginas usando as tags HTML não podem ser interpretadas para o reutilização das informações nelas contidas, pelo fato de não apresentarem o real significado semântico das informações presentes nas páginas. Observa-se então a indispensabilidade de abordar a estruturação de informações exibidas no sítio em formato RDF e/ou JSON, ou seja, a 
representação da estrutura dos elementos exibidos numa página HTML também no formato RDF e/ou JSON.

De forma similar, Marx et al. (2012) propõem um plugin extensível para o Integrated Development Environment (IDE) Eclipse, cujo objetivo é converter dados do RDB (Relational Database System) para o formato de arquivo RDF (Resource Description Framework), seguindo as recomendações do documento elaborado pela Consórcio World Wide Web (W3C) - R2RML: mapeamento linguagem RDB para RDF.

Baseando-se na apresentação de cenários nos estudos de caso realizados pelo W3C, é possível gerar a correspondência de um código de um documento HTML para RDF e JSON. Marx et al. (2012) dizem que devem ser encorajados os esforços para criação de algoritmos para correspondência automática de vocabulário e o fornecimento de suporte para dados relacionais em nuvem. Os autores destacam que a arquitetura proposta deve ser extensível e acolhedora para mudanças que aconteçam.

Portanto, considerar que cada página HTML apresentada no navegador terá um arquivo RDF e/ou JSON correspondente é o princípio básico do método proposto neste estudo. Ambos os formatos, RDF e JSON, devem ser disponibilizados juntamente com a publicação da página HTML para que outras ferramentas e/ou sistemas computacionais possam verificar e consumir as informações, utilizando o formato que julgar mais conveniente, e deste modo possam reutilizá-las por completo.

A manutenção constante das estruturas correspondentes pode garantir o monitoramento das informações publicadas, o compartilhamento, a análise $\mathrm{e}$ reutilização. Salienta-se que na revisão de literatura não foram encontrados estudos que abordassem a correspondência de página HTML com RDF e JSON, o que caracteriza singularidade no processo de publicação de informações usando o método TRADIN proposto, a seguir.

\section{Método TRADIN}

O método proposto neste trabalho está embasado na arquitetura de solução de integração proposta por Paiva, Revoredo e Baião (2016), conforme mostra a Figura 1, buscando uma publicação dos dados primários de forma direta, originados dos sistemas utilizados internamente pelo órgão governamental, eliminando etapas para publicação e distribuindo a responsabilidade na busca pela transparência.

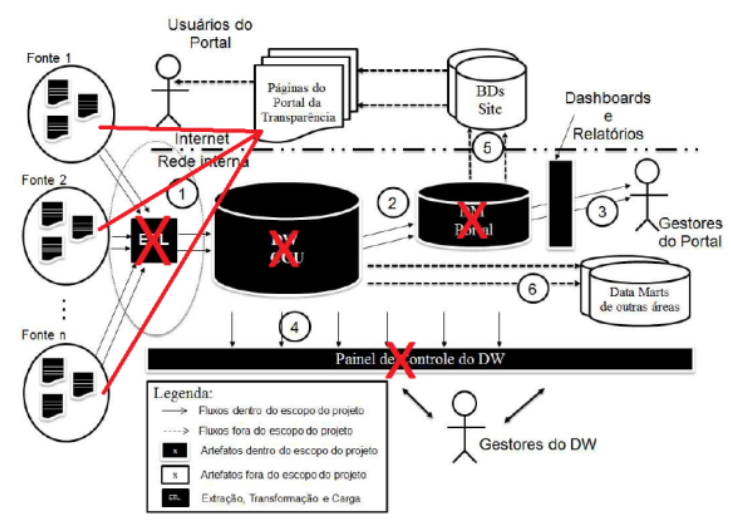

Figura 1. Arquitetura da solução de integração Paiva, Revoredo e Baião (2016) indicando as etapas excluídas com o método TRADIN. 
Reformulando a arquitetura proposta por Paiva, Revoredo e Baião (2016), nas fontes primárias de dados deverão ser disponibilizados os objetos de consulta que serão vinculados diretamente nas páginas do Portal de Transparência dos órgãos governamentais, ou seja, a construção da página será efetuada consumindo objetos de consultas presentes em diversos hosts, que, no entanto, serão reunidos numa publicação de página do portal. Os Dashboards e Relatórios deverão ser também individualizados nas fontes primárias, e da mesma forma que os objetos de consulta, serão reunidos na publicação da página no Portal Transparência.

Desse modo, a principal contribuição deste trabalho é a proposta do método TRADIN ilustrado na Figura 2. O método está dividido em duas principais visões sendo de um lado o órgão governamental e do outro lado os sítios, navegadores e sistemas computacionais diversos, como descrito a seguir.

O sistema administrativo do portal transparência faz a publicação das páginas no sítio do órgão governamental, compondo a página publicada de elementos vinculados as mídias HTML ou que fazem referência a objetos externos de outros sistemas. Em seguida, notifica os sítios que registraram marcação na página, "Sítios de Destino" (Figura 2). Esses sítios por sua vez também possuem o sistema de administração de publicação de páginas seguindo recomendações do método TRADIN, que importa as informações, caso o administrador do sítio de destino deseje consumir ou replicar aquelas informações recebidas. A página é publicada nos formatos correspondentes (HTML, RDF e JSON) para atender navegadores, sítios e outros sistemas computacionais presentes nesta visão.

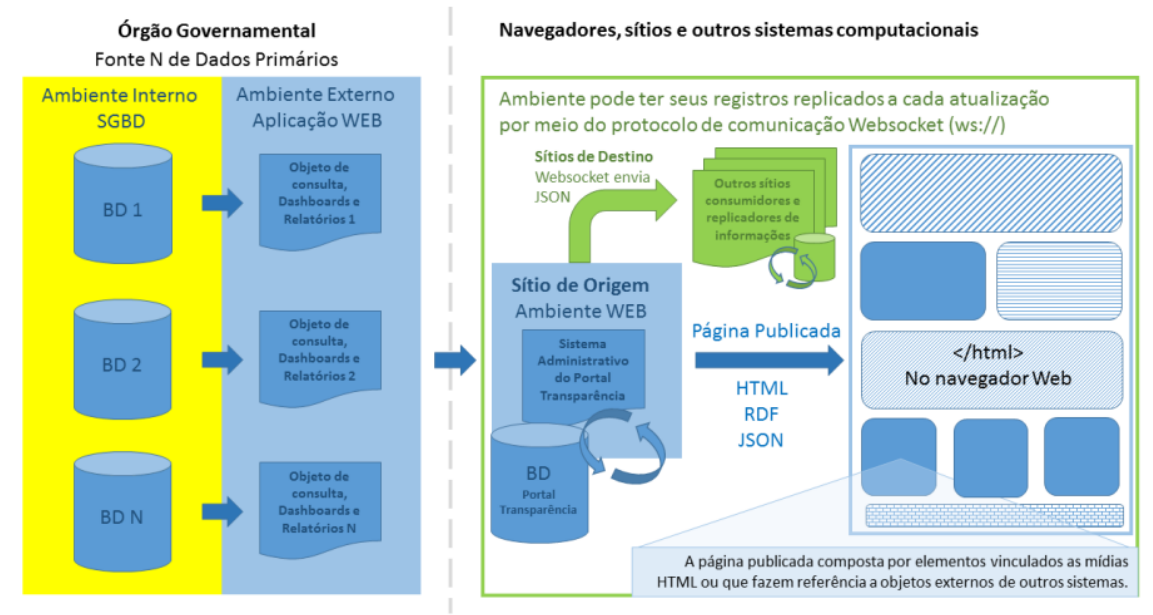

Figura 2. Arquitetura do Método Proposto.

A arquitetura do método TRADIN propõe a construção individual de objetos de consulta, dashboard e relatórios para cada SGBD (Sistema Gerenciador de Bando de Dados), criando $N$ fontes de dados primários, ou seja, publicados diretamente da fonte de trabalho. Na construção das páginas do Portal Transparência do órgão e no sistema administrativo do portal, serão vinculados os elementos que compõem a página, com suas mídias HTML e todas as referências das consultas provenientes do SGBD. A cada nova publicação na página, executa-se o evento Websocket que envia a mensagem com as informações da página para os sítios de destino cadastrados para receberem informações da referida página. A padronização das informações da página para a troca 
de mensagens é definida pelo sítio de origem (Portal Transparência) que deste modo não necessita ter conhecimento sobre os sítios de destino (clientes), bastando apenas comunicá-los.

O protocolo Websocket foi escolhido para a comunicação por tratar-se de um protocolo simplificado e ter uma camada sobre o TCP (Transmission Control Protocol), na qual se adicionou um modelo de segurança web baseada na origem e um esquema de nomenclaturas e endereços, que suportam a multiplicidade de serviços na mesma porta de comunicação e vários hosts no mesmo endereço IP [Varela e Loh 2012].

Registros de páginas, elementos e mídias HTML são mantidos no banco de dados, estabelecendo-se o vínculo das últimas com os elementos, em cadastros básicos que irão retornar, após o processamento por linguagem de programação, a saída do HTML no navegador web e dos arquivos RDF e JSON.

Ao gerar todos os formatos de saída (HTML, RDF e JSON), o sítio de origem captura o JSON gerado na publicação da página e envia para os sítios de destino cadastrados para receberem notificações da página específica. Para o envio da mensagem, abre-se uma porta de comunicação entre os servidores de aplicação por meio do protocolo Websocket usando a URI do sítio de destino, informada no momento do cadastro de marcação da página no sítio de origem.

A comunicação entre sítios é necessária e vai ao encontro dos anseios do cidadão e dos órgãos governamentais na administração dos recursos públicos. Trata-se de uma metodologia de compartilhamento, fiscalização e processamento de dados realizada por meio de diversos sítios, sejam eles públicos ou privados, obedecendo a um padrão de compartilhamento de conteúdo e dados para análise de forma direcionada. Deste modo, os diferentes segmentos da sociedade poderão acompanhar os dados publicados, com a possibilidade de seleção e registro para recebimento dos dados que são de interesse do cidadão, podendo replicar as informações em seus próprios sítios se assim desejarem.

O protocolo Websocket permite a abertura de um canal de comunicação sem depender da abertura de várias conexões HTTP, possibilitando a comunicação em duas vias entre um agente usuário e um host remoto, assim é possível prover um mecanismo pelo qual aplicações web possam se comunicar de forma bidirecional [Varela; Loh, 2012]. A cada atualização de página, o sítio de origem notifica o(s) sítio(s) de destino por meio do Websocket, o qual decide se incorpora a informação em seus registros, podendo optar pela plenitude ou parcialidade da informação, que vem por meio do JSON do sítio de origem.

\section{Conclusões}

Este estudo apresentou a concepção do método TRADIN como meio de propiciar ambiente adequado para publicação de informações na internet, com alta disponibilidade ao cidadão, escalabilidade de compartilhamento por meio do protocolo de comunicação escolhido, bem como pelos formatos de padronização da estrutura de publicação de informações.

Para validar o método proposto, foram realizados experimentos a partir da prospecção de cenários, a fim de medir o alcance e eficiência do método proposto neste 
estudo, de modo prático [Blind Review 2017]. Os experimentos realizados permitiram visualizar o método em relação ao dinamismo e integração dos dados.

Não há como acreditar que o Governo trabalhando sozinho, mesmo com sua grandiosidade, seja capaz de fornecer dados abertos ou realizar a integração dos diferentes dados de diversas fontes sistêmicas, quer seja por falta de interesse, alto custo de gestão ou falta de entendimento para a sua realização. O modelo público-privado mostra-se como uma solução eficiente, devido a amplitude dos atores envolvidos.

A utilização do método TRADIN possibilita ao órgão governamental maior facilidade no alcance do Nível 5 (examinável) do Modelo de Maturidade de Transparência Governamental de Cappelli et al. (2014), atendendo ainda aos oito princípios sobre Dados Governamentais Abertos [Davies 2013].

Pretende-se utilizar o padrão de integrabilidade definido pelo TRADIN por meio do protocolo Websocket para iniciar discussões a respeito dos benefícios de desempenho e o alcance da redução de custos de infraestrutura na disponibilização de serviços ao comparar a metodologia de integrabilidade proposta com REST (Representational State Transfer), o principal estilo arquitetural utilizado na atualidade.

Por fim, crê-se que este estudo representa uma importante contribuição para a área de Engenharia Web e Governo Eletrônico e áreas correlatas, que fazem uso de dados oriundos dos órgãos de governo. Em seu contexto geral, o método TRADIN apresenta um padrão alternativo para organização e reutilização das informações na Internet.

\section{Referências}

Araújo, C. L. B. e Maia, L. C. G. (2014) Gestão Pública e Governo Eletrônico: Estudo sobre Sítios institucionais de Prefeituras no Estado de Minas Gerais. Democracia Digital e Governo Eletrônico, Florianópolis/SC.

Bargh, M. S., Choenni, S., e Meijer, R. (2015) Privacy and Information Sharing in a Judicial Setting: A Wicked Problem. Proceedings of the 16th Annual International Conference on Digital Government Research, Arizona State University.

Batista, C. e Silva, C. (2015) Um Processo Criativo de Elicitação de Contextos para Sistemas Sensíveis ao Contexto. XI Simpósio Brasileiro de Sistemas de Informação (SBSI), Goiás.

Brasil. Constituição (1988). Constituição da República Federativa do Brasil. Texto consolidado até a Emenda Constitucional n $n^{\circ} 93$ de 08 de setembro de 2016. Brasília, DF: Senado Federal, $2016 . \quad$ DOI: http://www.senado.gov.br/atividade/const/con1988/con1988_08.09.2016/CON1988. pdf. Acesso em 19 de out 2016.

Cappelli, C., Engiel, P., Leite, J., Asteriotanaka, V., Santos, G. e Benjamim, K. (2014) A Evolução do Modelo de Maturidade em Transparência Organizacional. Simpósio Brasileiro de Sistemas de Informação (SBSI).

Collins-Williams, T. e Wolfe, R. (2010) Transparency as a trade policy tool: the WTO's cloudy windows. World Trade Review. University of Cambridge, United Kingdom. 
Cruz, W. G. S., Maciel, C., Girata, N. N. H. e Castilho, F. B. M. (2016) Um método quantitativo para avaliar a adoção de Dados Abertos nos Tribunais de Contas do Brasil. iSys - Revista Brasileira de Sistemas de Informação, Rio de Janeiro.

Davies, T. Open Data Barometer 2013 Global Report. World Wide Web Foundation and Open Data Instituto. 2013. Disponível em http://www.opendataresearch.org/ dl/ odb2013/Open-Data-Barometer-2013-Global- Report.pdf. Acesso 14 de nov. 2016.

Espíndola, C. E., Oliveira, J. B. F. e Formiga, M. M. (2011) A Tecnologia da Informação como meio para facilitar o acesso do Cidadão aos Serviços Públicos. IV Congresso Consad de Gestão Pública, Brasília/DF.

Klein, R. H., Luciano, E. M. e Macadar, M. A. (2015) Grau de Transparência de Dados Abertos Governamentais: o Portal Governamental Brasileiro dados.rs.gov.br. Twenty-first Americas Conference on Information Systems, Puerto Rico.

Martano, A. e Craveiro G. (2014) Abertura e Disponibilização de Dados Abertos Governamentais: Estudos de Caso. Simpósio Brasileiro de Sistemas de Informação (SBSI).

Marx, E., Salas, P., Breitman, K., Viterbo, J. e Casanova, M. A. (2012) RDB2RDF: A relational to RDF plug-in for Eclipse. Software: Practice And Experience. John Wiley \& Sons, Ltd.

Mendonça, P. G. A., Maciel, C. e Filho, J. V. (2015) Visualizing Aedes aegypti infestation in urban areas: a case study on open government data mashups. Information Polity, vol. 20, no. 2,3, p. 119-134.

Paiva, E., Revoredo, K. e Baião, F. (2016) DW-CGU: Integração dos Dados do Portal da Transparência do Governo Federal Brasileiro. iSys - Revista Brasileira de Sistemas de Informação, Rio de Janeiro.

Rek, M. (2013) Os princípios basilares da administração pública e os aplicáveis às licitações. In: Âmbito Jurídico, Rio Grande, XVI, n. 116, set 2013. DOI: http://www.ambitojuridico.com.br/site/?n_link=revista_artigos\%20_leitura\&artigo_i $\mathrm{d}=13610$. Acesso em 19 de out 2016.

Santos, R., Cappelli, C., Maciel, C. e Leite, J. C. S. P. (2016) Transparência em Ecossistemas de Software. VII Congresso Brasileiro de Software - Teoria e Prática (CBSoft).

Stake, R. E. (2011) Pesquisa Qualitativa: estudando como as coisas funcionam. Porto Alegre, RS: Artmed Editora S.A.

Varela, T. D. R. e Loh, S. (2012) Implementação E Análise Da Utilização De Websockets Em Sistemas Computacionais. Universidade Luterana do Brasil (ULBRA), Rio Grande do Sul.

Washington, A. L., Willis, D. e Tauberer, J. (2012) Do-it-yourself Transparency: Emerging methods of Congressional information dissemination. The proceedings of the 13th Annual International Conference on Digital Government Resource. College Park, MD, USA. 